\title{
INVESTIGATION OF LAYERED ORTHOTROPIC STRUCTURES BASED ON ONE MODIFIED REFINED BENDING THEORY
}

\author{
Kasimov A.T. ${ }^{1}$, Yessenbayeva G.A. ${ }^{2}$, Zholmagambetov S.R. ${ }^{1}$, Khabidolda O. ${ }^{2}$ \\ ${ }^{1}$ Karaganda Technical University, Karaganda, Kazakhstan \\ 2Buketov Karaganda University, Karaganda, Kazakhstan, esenbaevagulsima@mail.ru
}

\begin{abstract}
In the article, constructions made of orthotropic multilayer composite material, in particular, layered orthotropic plates are considered. Numerical modeling and analysis of the stress-strain state for the plates are carried out on the basis of one version for the refined theory of layered plates. The bending problems for plates of medium thickness and thin multilayer plates of symmetric and asymmetric structures are investigated. All studies are conducted taking into account the properties of orthotropy and multilayering for the composite material from which the plates are made. The general algorithm for the numerical calculation of the stress-strain state for layered plates with orthotropic layers is developed on the basis of the finite difference method. This algorithm is implemented on a PC by a software package.
\end{abstract}

Keywords: orthotropic materials, layered composites, layered (or laminated) orthotropic plate, refined bending theory, transverse shear, deflection, strain, asymmetry, multilayer structure, finite difference method

\section{Introduction}

The design of new modern technique and the improvement of technological developments made it necessary to search and create new materials. Composite materials, in particular, multilayer composites, are a new type of such materials with a wide range of operational properties that cannot be achieved using traditional materials. The use of multilayer composite materials in modern apparatuses and devices required taking into account their structural features, physical properties of the materials used, as well as the creation of new methods for calculating the stress-strain state of such structures.

The rapid development of scientific and technological progress requires the creation and implementation of new progressive materials and structures with predetermined properties. Orthotropy is one of these properties. Orthotropic materials are more difficult to analyze than isotropic materials because their properties depend on the direction. In orthotropic materials there are two or three mutually perpendicular axes of symmetry, with respect to which the material properties differ significantly. For example, the properties of wood material along and across the fibers are very different.

Reinforced concrete (with different reinforcement stiffness in mutually orthogonal directions $x$ and $y$ ), many polymers (plastics, etc.), wood, metal sheets after rolling or upsetting, composites with two families of threads, layered composites, etc. belong to orthotropic materials. Layered or multilayer composite materials consist of alternating layers of filler and matrix material. This design is often called a package. The design of layered composite structures usually contains separate layers of different materials, connected together and combined into one structure. A layered composite structure has properties that none of its layered components can possess individually.

In multilayer composite structures, the layers are made of such a material and these layers are arranged so as to endow the structure with a number of predetermined positive properties. At the same time, the materials are selected in such a way that, in an optimal combination, they give a qualitatively new type of construction. Or, in other words, in multilayer composite structures, the layers are arranged so that, under operational conditions, the structure better corresponds to its functional purpose. Layered composite structures include layered (or laminated) orthotropic plates.

Layered orthotropic plates are increasingly widely used in various fields of technique, mechanical engineering and construction. The interest in layered plates is primarily due to the fact that they have a set of properties and features that qualitatively distinguish them from traditional structures made of homogeneous materials. The combination of layers in the material makes it possible to obtain a structure that combines high strength and stiffness with relatively low weight and high technical and operational requirements. 
In practice, structures made of three-layer material are most widespread. Under operating conditions, such materials are the most rational from the point of view of ensuring a minimum for weight indicators with the required strength and stiffness. But they do not always meet all the requirements for building structures and elements of modern technology. The technical, physical and mechanical properties of structures made of multilayer inhomogeneous materials differ significantly in the thickness of their packages. Therefore, the features study for the operation of structures made of multilayer inhomogeneous materials in the thickness of their package by use refined models is important in the design of new innovative lightweight structures made of multilayer materials. Multilayer plate theories that refine the technical theory should take into account the strain in the transverse direction of the material and the factors associated with it.

The specific features of soft layers of material in multilayer structures, the joint work of the package layers under the influence of external loads and the low resistance of the filler material in the transverse direction give rise to a variety of existing theories [1-12]. Currently, the theory of calculation for multilayer structures is rapidly developing and improving. However, in practice, when solving specific problems, difficulties associated with features of multilayer structures arise.

It should be noted that analytical results for structures made of multilayer composite material were obtained for a limited number of problems. Therefore, approximate numerical methods, the implementation of which opens up great opportunities for researchers, began to be widely used.

The assessment of the stress-strain state for such multilayer systems is a difficult problem, and in practice it is not always possible to obtain their solution in a closed form. In this regard, it becomes necessary to develop an effective numerical calculation, based on a refined theory, which makes it possible to determine and investigate the components of the stress-strain state for the package layers in an automated mode.

\section{Initial positions, hypotheses, and the plate model}

We consider a rectangular layered plate with sides $a_{1}$ and $a_{2}$ (fig. 1), with orthotropic layers and a thickness $H=\delta_{1}+\delta_{2}$, consisting of an arbitrary number of orthotropic layers. We consider a plate in an orthogonal coordinate system $x_{1}, x_{2}, x_{3}=z$. The axes $x_{1}$ and $x_{2}$ lie on the coordinate plane and their directions coincide with the orthotropy axes of the layers. The coordinate plane is positioned arbitrarily along the height of the plate cross-section. The distance from the coordinate plane to the lower and upper surfaces of the plate is denoted, respectively, by $\delta_{1}$ and $\delta_{2}$.

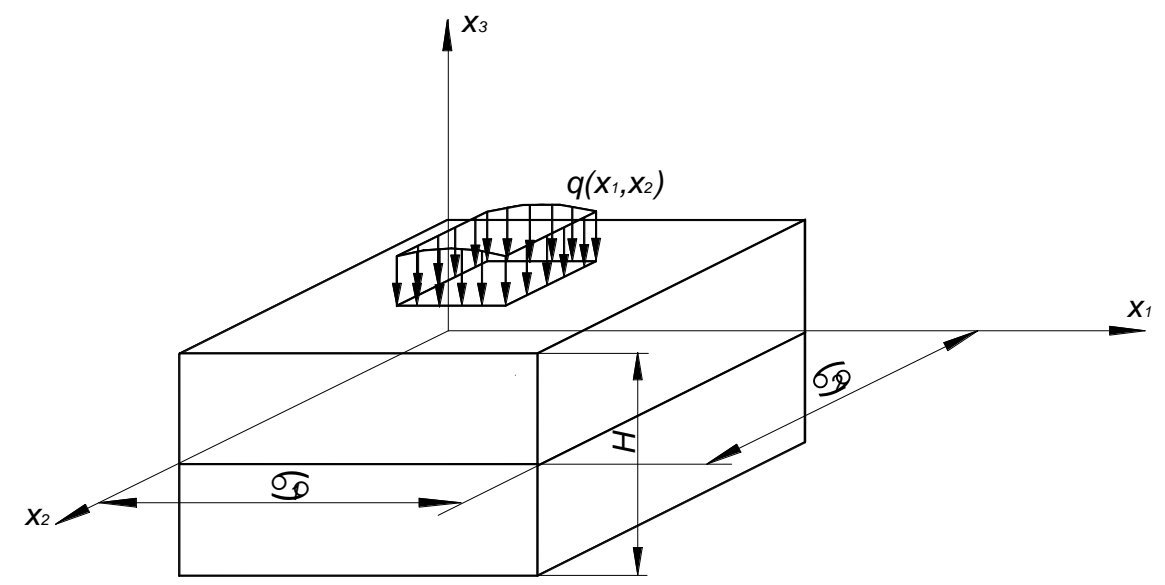

Fig.1. Calculation scheme of the plate

The material layers are numbered from the bottom surface of the plate (fig. 2). The total number of layers in the package is denoted by $n$, then we take $k=1,2, \ldots, n$, where $k$ is the number of an arbitrary layer. The layers in which the coordinate surface is located will be denoted by $\mathrm{m}$. All layers of the plate in aggregate in thickness form a package of layers. 
In the general case, we assume that the structure of the package is formed by layers of different thickness and stiffness, the physical and mechanical characteristics of which are constant in their thickness. The number and order of the layers are arbitrary.

We assume that at the boundary when passing from layer to layer, the static and kinematic conditions are fulfilled. This corresponds to the operation of their layers without slipping and tearing.

Let a normal load $q\left(x_{1}, x_{2}\right)$ act on the upper surface of the plate. The normal load $q\left(x_{1}, x_{2}\right)$ varies according to an arbitrary law. The positive direction of the normal load coincides with the direction of the normal axis $x_{3}=z$.

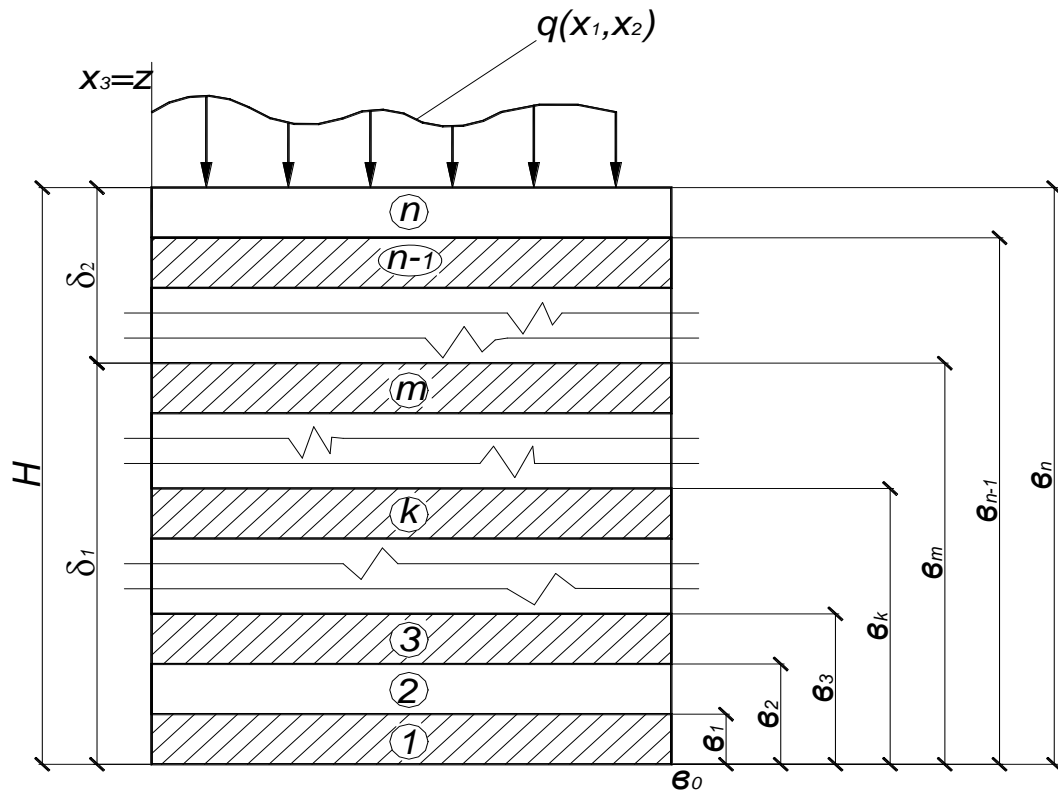

Fig. 2. The cross section of the layered plate

On the plate surface, the boundary conditions take the form

$$
\sigma_{33}^{n}=q\left(x_{1}, x_{2}\right), \quad \sigma_{i 3}^{n}=0, \quad \sigma_{i 3}^{1}=0, i=1,2,3 .
$$

As the main assumptions for constructing a new refined model of the stress-strain state for a layered plate of an asymmetric structure with orthotropic layers, we accept the following system of hypotheses

$$
\sigma_{i 3}^{k}=G_{i 3}^{k} \psi_{i, 3}^{k}(z) \chi,_{i} ; \quad \sigma_{33}^{k}=-\sum_{i=1}^{2} \eta_{3 i}^{k}(z) \chi,_{i i} ; \quad u_{3}^{n}=W .
$$

The given hypotheses are obtained on the basis of the hypotheses proposed by professor A.Sh.Bozhenov [1], by neglecting a number of factors that insignificantly affect the stress-strain state of plates.

Here $W$ и $\chi$ are sought functions of the deflection and shear of the coordinate surface, depending on the coordinates $x_{1}$ and $x_{2} . G_{i 3}^{k}$ is the shear modulus of the material for the $k$ th layer of the plate, remaining components are distribution functions that depend on the transverse coordinate $z$.

Hypotheses (1) satisfy the conditions for joint operation of layers without separation and displacement, as well as conditions on the plate surfaces and determine the nonlinear law of variation of transverse shear stresses and normal transverse stresses in the plate thickness. It is assumed that normal displacements are equal to deflections.

For the distribution function in expressions (1), we have the following formulas

$$
\begin{array}{r}
\psi_{i, 3}^{k}(z)=\frac{1}{G_{i 3}^{k}}\left[\eta_{2 i}^{k}(z)-\eta_{1 i}^{k}(z) \delta_{i}^{*}\right] ; \quad \eta_{1 i}^{k}(z)=\int_{b_{k-1}-\delta_{1}}^{z} A_{i}^{k} d z+\sum_{j=1}^{k-1} \int_{b_{j-1}-\delta_{1}}^{b_{j}-\delta_{1}} A_{i}^{j} d z ; \\
\eta_{2 i}^{k}(z)=\int_{b_{k-1}-\delta_{1}}^{z} B_{i}^{k} z d z+\sum_{j=1}^{k-1} \int_{b_{j-1}-\delta_{1}}^{b_{j}-\delta_{1}} B_{i}^{j} z \cdot d z ; \quad \eta_{3 i}^{k}(z)=\int_{b_{k-1}-\delta_{1}}^{z} G_{i 3}^{k} \psi_{i, 3}^{k}(z) d z+C_{3 i}^{k} ;
\end{array}
$$

where the constants have the form 


$$
C_{3 i}^{k}=\sum_{j=1}^{k-1} \int_{j-1}^{b_{j}-\delta_{1}} G_{i 3}^{j} \psi_{i, 3}^{j}(z) d z .
$$

Here and in what follows, the notation introduced in [1] is adopted. For the components in formulas (2) and (3), we have the following expressions

$$
\begin{gathered}
A_{i}^{k}=0,5\left\{B_{i i}^{k}\left(1+v_{i e}^{k}\right)+G_{12}^{k}\right\} ; \quad B_{i}^{k}=0,5 B_{i i}^{k}\left(1+v_{i e}^{k}\right)+G_{12}^{k} ; \quad B_{i i}^{k}=E_{i}^{k} v_{0}^{k} ; \\
\delta_{i}^{*}=\eta_{2 i}^{k} / \eta_{1 i}^{k} ; \quad v_{0}^{k}=\left(1-v_{12}^{k} v_{21}^{k}\right)^{-1} ; \quad B_{i 3}^{k}=\left(v_{3 i}^{k}+v_{l i}^{k} v_{3 l}^{k}\right) v_{0}^{k} .
\end{gathered}
$$

Based on the accepted hypotheses (1), we construct a linear geometric model of a layered plate and establish a relationship between stresses and strains.

\section{Development of a bending model for layered orthotropic plates with an asymmetric structure in the thickness}

To construct a linear geometric model of a layered orthotropic plate and establish a relationship between stresses and strains, we use some well-known relations for the three-dimensional theory of elasticity.

At the same time, we will take into account that for an arbitrary kth layer of the plate, simplified hypotheses are adopted, they satisfy the conditions for the joint operation of the layers without separation and displacement, as well as the conditions on the surface of the plate, and determine the nonlinear law of variation of transverse shear stresses and normal stresses in the plate thickness. Factors such as a transverse shear in two directions and pressure of layers on each other, as well as orthotropy of layers, will be taken into account by one shear function.

We determine the transverse shear strain from Hooke's law by substituting the hypothesis expression for transverse tangential stresses (1). We find normal transverse strains from the last Cauchy relation taking into account (1).

From the third Cauchy relation, after integrating over $z$ and taking into account formulas (1) and formulas for the transverse shear strain, we obtain an expression for tangential displacements. Normal displacements are considered equal to deflections. The distribution functions are determined from the conditions for the contact of the layers and from the conditions on the coordinate surface.

Tangential strains are determined from the first Cauchy relations, substituting expressions for tangential displacements in them. Taking into account formulas (1) and expressions for tangential strains, the stress are found from the generalized Hooke's law.

Received expressions of displacements and strains allow us to construct a geometric model of multilayer orthotropic plates and determine the components of the stress-strain state at an arbitrary point in the kth layer. Within the framework of the accepted hypotheses, all equations of the elasticity theory are approximately satisfied. Thus, the constructed new model describes the three-dimensional law of the change in the stress-strain state of multilayer plates with an asymmetric structure in thickness. The model is twodimensional, since all the sought functions are functions of the coordinate surface.

Equations for the bending multilayer orthotropic plates with an asymmetric structure in thickness are obtained from the Lagrange variational principle using the relations received on the basis of the accepted hypotheses. Then, by introducing the force functions, the system of equations and the boundary conditions are transformed into a mixed form. As a result, a system of three equations of the 12th order is obtained; this system describes the bending for a multilayer plate of an asymmetric structure in thickness with orthotropic layers. The system of resolving equations of a layered plate is presented in a transformed form in [1] and in a mixed form this system takes the following form

$$
\begin{gathered}
\Delta_{F}^{2} \phi+\Delta_{1 S}^{2} W-\left(\Delta_{2 S}^{2}-\Delta_{13}^{2}\right) \chi=0 ; \\
\Delta_{1 S}^{2} \phi+\left(\Delta_{3 S}^{2}-\Delta_{D}^{2}\right) W+\left(\Delta_{P}^{2}-\Delta_{23}^{2}-\Delta_{4 S}^{2}\right) \chi=-q \\
\Delta_{2 S}^{2} \phi+\left(\Delta_{5 S}^{2}-\Delta_{p}^{2}\right) W+\left(\Delta_{P 1}^{2}-\Delta_{33}^{2}-\Delta_{P 3}\right) \chi=0 .
\end{gathered}
$$

The system takes into account a transverse shear, a layer pressure, and normal strains. Three functions of the coordinate surface are unknown; these functions are the function of force, the deflection function, and the shear function. We have the following relations 


$$
\begin{gathered}
\Delta_{f}^{2}=A_{1}^{*}()_{, 1111}+A_{2}^{*}()_{, 1122^{*}}+A_{3}^{*}()_{, 2222} ; \\
\Delta_{g}=B_{1}^{*}\left({ }_{-11}+B_{2}^{*}\left({ }_{, 2}\right)_{, 22} \cdot\right.
\end{gathered}
$$

Equations (4) contain differential operators of the 4th and 2nd orders, which are determined from (5) with coefficients, respectively, $A_{j}^{*}(j=1,2,3)$, and $B_{i}^{*}(i=1,2)$, depending on the stiffness of the layered plate. For different values of $f$ and $g$, the coefficients of the operators take different values, which are shown in Table 1.

Table 1. Coefficients of differential operators

\begin{tabular}{|c|c|c|c|c|c|c|}
\hline \multirow{2}{*}{ Value of $f$} & \multicolumn{3}{|c|}{ Coefficients } & \multirow{2}{*}{ Value of $g$} & \multicolumn{2}{c|}{ Coefficients } \\
\hline & $\mathrm{A}_{1}{ }^{*}$ & $\mathrm{~A}_{2}{ }^{*}$ & $\mathrm{~A}_{3}{ }^{*}$ & & $\mathrm{~B}_{1}{ }^{*}$ & $\mathrm{~B}_{2}{ }^{*}$ \\
\hline $\mathrm{F}$ & $\mathrm{F}_{1}$ & $\mathrm{~F}_{4}-2 \mathrm{~F}_{2}$ & $\mathrm{~F}_{3}$ & $\mathrm{P}_{3}$ & $\mathrm{P}_{13}$ & $\mathrm{P}_{23}$ \\
\hline $1 \mathrm{~S}$ & $\mathrm{R}_{1}$ & $\mathrm{R}_{2}+\mathrm{R}_{3}-\mathrm{R}_{5}$ & $\mathrm{R}_{4}$ & & & \\
\hline $2 \mathrm{~S}$ & $\mathrm{I}_{1}$ & $\mathrm{I}_{2}+\mathrm{I}_{3}-\mathrm{I}_{5}$ & $\mathrm{I}_{4}$ & & & \\
\hline 13 & $\mathrm{H}_{1}$ & $\mathrm{H}_{2}$ & $\mathrm{H}_{3}$ & & & \\
\hline $\mathrm{D}$ & $\mathrm{D}_{11}$ & $2\left(\mathrm{D}_{12}+2 \mathrm{D}_{66}\right)$ & $\mathrm{D}_{22}$ & & & \\
\hline $3 \mathrm{~S}$ & $\mathrm{RK}_{1}$ & $\mathrm{RK}_{2}$ & $\mathrm{RK}_{3}$ & & & \\
\hline $4 \mathrm{~S}$ & $\mathrm{IK}_{1}$ & $\mathrm{IK}_{2}$ & $\mathrm{IK}_{3}$ & & & \\
\hline 23 & $\mathrm{C}_{14}$ & $\mathrm{C}_{14}+\mathrm{C}_{24}$ & $\mathrm{C}_{24}$ & & & \\
\hline $\mathrm{P}$ & $\mathrm{P}_{11}$ & $\mathrm{P}_{122}^{\prime}$ & $\mathrm{P}_{222}$ & & & \\
\hline $5 \mathrm{~S}$ & $\mathrm{I}_{1}^{\prime}$ & $\mathrm{I}_{2}^{\prime}+\mathrm{I}_{3}^{\prime}+\mathrm{I}_{5}^{\prime}$ & $\mathrm{I}_{4}^{\prime}$ & & & \\
\hline $\mathrm{P}_{1}$ & $\mathrm{~S}_{111}$ & $\mathrm{~S}_{122}^{\prime}$ & $\mathrm{S}_{222}$ & & & \\
\hline 33 & $\mathrm{C}_{15}$ & $\mathrm{C}_{15}^{\prime}+\mathrm{C}_{25}$ & $\mathrm{C}_{25}$ & & & \\
\hline
\end{tabular}

We note that the solution of the obtained systems of equations is possible when six boundary conditions are satisfied on each contour with respect to the sought functions.

\section{Numerical study of the stress-strain state for layered orthotropic plates}

The discretization of the system of resolving equations and their corresponding contour conditions [2] is performed by the finite difference method (FDM) in a rectangular grid. In a compact matrix form, a method for the group exclusion of the unknown functions at the contour points for the grid domain of the plate has been developed. Based on the finite difference method, a general algorithm for numerical calculation of the stress-strain state of layered plates with orthotropic layers of arbitrary structure in thickness is developed and implemented on a PC by a software package. This software package contains a head program and several subroutines implemented in the FortRUN language. The flowchart of the head program consists of several blocks, each of which is a standalone module and performs certain functions. A brief explanation is given below for these blocks.

Block 1. Input of the initial parameters. For ease of calculation, all dimensional values are given in dimensionless form.

Block 2. Finding the stiffness characteristics for a layered plate.

Block 3. Drawing up and solving the system of the equilibrium equation for a layered plate.

Block 4. Finding the stress-strain state of a layered plate.

The solution for the obtained systems of equations is possible when six boundary conditions on each contour are satisfied with respect to the sought functions.

If we turn to history, it is easy to recall that fiberglass and carbon fiber were among the first elements of layered composites. However, this type of composite structures, qualitatively transformed and widely used in construction, still needs development and research.

On the basis of the constructed new two-dimensional model according to the proposed numerical method, the study of the bending problem for hinge-supported, symmetric in thickness, square plates with orthotropic layers of carbon fiber in a wide range of variations for $h / a$ is performed. These plates have the following physical, mechanical and geometric characteristics for the corresponding layers of the plates:

- the elastic modulus of the plate layers: $E_{1}=25 E_{2}, E_{2}=E_{3}$;

- the shear modulus of the plate layers: $G_{12}=G_{13}=0.5 E_{2}, G_{23}=0.2 E_{2}$; 
- Poisson's ratio of the plate layers: $v_{12}=v_{23}=v_{13}=v_{32}=0.25, v_{21}=v_{31}=0.01$;

- the plate layer thickness: $h_{1}=h_{3}=0.5 h, h_{2}=0.25 h$.

We recall that the elasticity coefficients characterize the physical ability of a material (substance) to deform elastically when a force is applied to it. The elastic modulus or Young's modulus $(E)$ characterizes the physical property of the material, namely, the resistance of the material to tension / compression during elastic deformation. The shear modulus $(G)$ characterizes the ability of a material to resist shear deformation, that is, it determines the ability of a material to resist a change in shape while maintaining its volume. Poisson's ratio $(v)$ is the ratio of the relative transverse compression to the relative longitudinal tension. This coefficient does not depend on the size of the body, but on the nature of the material from which the structure is made. In Table 2, the presented values of deflections and tensile stresses in the center of the plate are compared with the results of known solutions according to the classical theory and to the methods presented in [3] and [4]. $\sigma_{11}$ is a tensile stress in the outer fiber of the lower layer, $\sigma_{22}$ is a tensile stress at the border of the outer and inner layers.

Table 2. Values of deflections and tensile stresses in the plate center

\begin{tabular}{|c|c|c|c|c|c|c|c|c|c|}
\hline$h / a$ & $\begin{array}{c}\text { Classical } \\
\text { theory }\end{array}$ & \multicolumn{2}{|c|}{ Exact solution [3] } & \multicolumn{2}{c|}{$\begin{array}{c}\text { Solution in } \\
\text { trigonometric series [4] }\end{array}$} & \multicolumn{2}{c|}{$\begin{array}{c}\text { Solution according to the } \\
\text { proposed method (FDM) }\end{array}$} & \multicolumn{2}{c|}{ Calculation error } \\
\cline { 2 - 11 } & $10^{-7} w, \mathrm{~m}$ & $\begin{array}{c}10^{-7} \mathrm{w} \\
\mathrm{m}\end{array}$ & $\frac{\sigma_{11}}{\sigma_{22}}, \mathrm{mPa}$ & $\begin{array}{c}10^{-7} \mathrm{w}, \\
\mathrm{m}\end{array}$ & $\frac{\sigma_{11}}{\sigma_{22}}, \mathrm{mPa}$ & $10^{-7} w, \mathrm{~m}$ & $\frac{\sigma_{11}}{\sigma_{22}}, \mathrm{mPa}$ & $w, \%$ & $\frac{\sigma_{11}}{\sigma_{22}}, \%$ \\
\hline $1 / 20$ & 690.00 & 820.41 & $\frac{217.20}{123.48}$ & 821.51 & $\frac{217.44}{123.48}$ & 840.56 & $\frac{209.08}{118.23}$ & 2.45 & $\frac{3.6}{4.25}$ \\
\hline $1 / 10$ & 43.12 & 73.70 & $\frac{55.90}{40.10}$ & 73.93 & $\frac{56.22}{40.26}$ & 75.85 & $\frac{53.95}{38.46}$ & 2.9 & $\frac{3.4}{4.1}$ \\
\hline $1 / 5$ & 2.70 & - & - & 9.25 & $\frac{16.59}{15.01}$ & 9.56 & $\frac{15.26}{14.85}$ & - & - \\
\hline $1 / 4$ & 1.10 & 4.96 & $\frac{11.52}{10.61}$ & 5.00 & $\frac{11.97}{10.96}$ & 5.15 & $\frac{11.48}{10.19}$ & 3.8 & $\frac{3.47}{3.96}$ \\
\hline $1 / 2$ & 0.07 & 0.81 & $\frac{5.55}{3.34}$ & 0.80 & $\frac{5.57}{3.39}$ & 0.85 & $\frac{5.32}{3.21}$ & 4.94 & $\frac{4.14}{3.29}$ \\
\hline
\end{tabular}

A comparison of the results obtained by the proposed numerical method with the three-dimensional exact solution [3] and the solution [4] in the entire range of the considered parameters $h / a$ shows a sufficiently acceptable accuracy of the numerical method for solving plate bending problems. The calculation results presented in Table 2 also show that the classical theory is not applicable to the problems under consideration.

A comparison for the results of applying the trigonometric series method and the numerical method proposed in the article shows that the results of solving by trigonometric series are more accurate. But here we note two important points.

Firstly, the trigonometric series method is an analytical method. Therefore, this method is more laborious and takes more time than the proposed numerical method implemented on a PC by a software package.

Secondly, the trigonometric series method can be used only for a limited range of plate bending problems, namely, in the presence of hinged support at the plate boundaries. The developed method of numerical calculation for layered orthotropic plates with arbitrary structure in thickness allows us to solve a wider range of problems with varying different boundary conditions, the geometric plate dimensions, the external loads, the thickness of the layers, and their elastic characteristics.

When assessing the reliability of the solutions obtained by the proposed method, the results of an experimental study for the bending of three-layer plates [5] are of great interest. The experiment was carried out on plates, the bearing layers of which were made of duralumin. The elastic modulus equals $E_{1}=E_{2}=7 \cdot 10^{5} \mathrm{~kg} / \mathrm{cm}^{2}$, Poisson's ratio is $v_{1}=v_{3}=0.32$, the layer thickness is equal to $0,081 \mathrm{~cm}$. The filler of the plate was plastic foam, in this case the shear modulus is equal to $G_{2}=890.7 \mathrm{~kg} / \mathrm{cm}^{2}$ and the Poisson's 
ratio is $v_{2}=0.4$. The bending of the plate was considered under a uniformly distributed load. So the problems were solved according to the proposed method for a number of plates under the action of a uniformly distributed load. In the experiment, the maximum deflections were measured. In Table 3, the results obtained are compared with experimental data.

Table 3. Comparison with experimental data for three-layer plates

\begin{tabular}{|c|c|c|c|c|c|c|c|}
\hline \multirow[b]{2}{*}{$\mathrm{N}$} & \multicolumn{3}{|c|}{ Plate parameters } & \multirow[b]{2}{*}{$\begin{array}{l}\mathrm{Load} q \\
\left(\mathrm{~kg} / \mathrm{cm}^{2}\right)\end{array}$} & \multicolumn{2}{|c|}{ Deflections, cm } & \multirow[b]{2}{*}{$\Delta \%$} \\
\hline & $a / h$ & $\begin{array}{c}\text { Side length } \\
a=b(\mathrm{~cm})\end{array}$ & $\begin{array}{l}\text { Filler thickness } \\
(\mathrm{cm})\end{array}$ & & Experiment [5] & $\begin{array}{c}\text { Obtained } \\
\text { solution }\end{array}$ & \\
\hline 1 & 76.5 & 112.3 & 1.306 & 0.038 & 0.4267 & 0.4307 & 0.93 \\
\hline 2 & 97.4 & 112.3 & 0.991 & 0.0507 & 0.9119 & 0.9401 & 3.09 \\
\hline 3 & 67.36 & 96.6 & 1.272 & 0.0507 & 0.3124 & 0.3258 & 5.56 \\
\hline 4 & 85.49 & 96.6 & 0.968 & 0.0456 & 0.4801 & 0.4901 & 2.08 \\
\hline 5 & 56.14 & 81.4 & 1,268 & 0.0507 & 0.1829 & 0.1701 & 6.9 \\
\hline 6 & 71.4 & 81.4 & 0.978 & 0.0507 & 0.2845 & 0.2760 & 2.98 \\
\hline 7 & 49.44 & 71.2 & 1.278 & 0.0406 & 0.0889 & 0.0855 & 1.9 \\
\hline 8 & 62.02 & 71.2 & 0.986 & 0.0507 & 0.1651 & 0.162 & 1.7 \\
\hline 9 & 38.09 & 55.8 & 1.303 & 0.1268 & 0.1168 & 0.1055 & 9.62 \\
\hline 10 & 48.4 & 55.8 & 0.981 & 0.0761 & 0.1120 & 0.0995 & 11.11 \\
\hline
\end{tabular}

Comparison of the results from Table 3 shows that the differences in deflection values are insignificant and for a large number of plates does not exceed $6 \%$.

\section{Conclusion}

In the article a method for numerical calculation of the stress-strain state for structures made of orthotropic, layered composite materials based on one modified refined bending theory is presented. When studying the stress-strain state of a structure, it is necessary to take into account the structure material, the structure texture, boundary conditions, forces and loads acting on the structure, etc. The physical properties of the material from which the structure is made and the material texture are taken into account by the research algorithm. Thus, in hypotheses (1), the conditions for the joint work of layers without separation and displacement are formulated, and the solution of problems is carried out on layers of multilayer structures, taking into account the interaction of layers. The physical properties of the material from which the structure is made are also taken into account by the characteristics of the material, in particular, the elasticity coefficients $(E, G, v$, etc.), which are present in the basic relations and equations used.

Comparisons of the results according to the proposed method with known solutions (Table 2) and experimental data (Table 3) indicate the reliability and greater universality of the developed method for the numerical calculation of the stress-strain state for layered orthotropic structures based on one modified refined bending theory.

\section{REFERENCES}

1 Bozhenov A.Sh. Theory of multilayer inhomogeneous plates, orthotropic shells and plates. Abstract of the dissertation of the doctor of technical sciences, Novosibirsk, 1990, 45 p. [in Russian]

2 Kasimov A.T. Investigations of the stress-strain state for rectangular multilayer plates by the finite difference method. Proceedings of the University, 2002, No. 4, pp. 73 - 75. [in Russian]

3 Pagano N.J., Hatflied S.J. Elastie behhavior of multilayered bidirectional composites. American Institute of Aeronautics and Astronautica Journal, 1972, Vol. 10, No. 7. p. 931 - 933.

4 Gorodetsky A.S., Zavoritsky V.I., Lantukh-Lyashchenko A.I., Razkazov A.O. Automation of calculations of transport facilities. Moscow, 1989, 232 p. [in Russian]

5 Alexandrov A.Ya., Bryukker L.E., Kurshin L.M., Prusakov A.P. Calculation of three-layer panels. Leningrad, 1960, 272 p. [in Russian]

6 Grigolyuk E.I., Kulikov G.M. Ways of developing the theory of elastic multilayer plates and shells. Bulletin of $T S T U$, 2005, Vol. 11, No. 2A, pp. 439 - 448. [in Russian]

7 Annin B.D., Volchko Yu.M. Non-classical models of the theory of plates and shells. Applied Mechanics and Technical Physics, 2016, Vol. 57, No. 5, pp. 5 - 14. [in Russian] 
8 Kasimov A.T. To the application of FDM for the calculation of layered plates taking into account shear strains along its thickness. Proceedings of the University, 2018, No. 3, pp. 100 - 103. [in Russian]

9 Piskunov V.G., Rasskazov A.O. Development of the theory of layered plates and shells. Applied Mechanics, 2002, Vol. 38, No. 2, pp. 22 - 28. [in Russian]

10 Bakulin V. Methods for optimal design and calculation of composite structures. Moscow, 2008, Vol.1, 256 p. [in Russian]

11 Popov B.G. Calculation of multilayer structures by variational matrix methods. Moscow, MSTU, 1993, 294 p. [in Russian]

12 Alfutov N.A., Zinoviev P.A., Popov B.G. Calculation of multilayer plates and shells made of composite materials. Moscow, 1984, 264 p. [in Russian] 